\title{
Optimization of engineering tolerance design using revised loss functions
}

\author{
Jeh-Nan $\operatorname{Pan}^{\mathrm{a}}$ and Jianbiao $\operatorname{Pan}^{\mathrm{b}}$ \\ ${ }^{a}$ Department of Statistics, National Cheng-Kung University, Tainan, Taiwan; ${ }^{b}$ Department of Industrial \\ and Manufacturing Engineering, California Polytechnic State University, San Luis Obispo, USA
}

\begin{abstract}
Engineering tolerance design plays an important role in modern manufacturing. Both symmetric and asymmetric tolerances are common in many manufacturing processes. Recently, various revised loss functions have been proposed for overcoming the drawbacks of Taguchi's loss function. In this article, Kapur's economic tolerance design model is modified and the economic specification limits for both symmetric and asymmetric losses are established. Three different loss functions are compared in the optimal symmetric and asymmetric tolerance design: a revised Taguchi quadratic loss function, an inverted normal loss function and a revised inverted normal loss function. The relationships among the three loss functions and process capability indices are established. A numerical example is given to compare the economic specification limits established by using the three loss functions. The results suggest that the revised inverted normal loss function be used in determining economic specification limits.
\end{abstract}

Keywords: symmetric and asymmetric tolerance design; Taguchi's quadratic loss function; inverted normal loss function; revised inverted normal loss function

\section{Introduction}

Product quality is highly regarded in today's business environment. Generally speaking, quality characteristics can be divided into three types: the 'nominal the best', the 'larger the better' and the 'smaller the better'. In the traditional quality evaluation system only the total cost to the producer was considered and minimized. A product is determined to be non-conforming if the quality characteristic of a product fails to meet the engineering specification limits defined by the producer. Taguchi (1986) argued that the total cost to the user should be considered as well, since a poorly designed product causes society losses from the initial design stage to the product usage. He defined the loss function as the deviation from the target/nominal quality characteristic. 
Taguchi's (1986) loss function has been extensively used in determining engineering tolerance (Kapur and Wang 1987, Kapur 1988, Kapur and Cho 1994). The drawbacks of Taguchi's quality loss function are that it is unbounded and symmetrical (Tribus and Szonyi 1989, Leon and $\mathrm{Wu}$ 1992). Currently various revised loss functions have been proposed for overcoming the drawbacks of the Taguchi loss function. For a detailed literature review of loss functions see Pan (2007).

In order to minimize total loss to the customer as well as to the producer, Kapur and Wang (1987), Kapur (1988) and Kapur and Cho $(1994,1996)$ proposed several optimization models for determining specification limits. Three costs were considered: inspection costs, scrap/rework costs and loss due to variation. Assuming that the quality characteristic $Y$ follows a normal distribution, Kapur's (1988) economic model can be written as

$$
T C=L_{\mathrm{Q}}^{\prime}+(1-q) \times S C+I C
$$

where $T C$ is the total expected losses per unit product, $L_{\mathrm{Q}}^{\prime}$ is the expected loss per unit product shipped to the customer, $S C$ is the scrap cost per unit, $I C$ is the inspection cost per unit and $q$ is the fraction of good products actually shipped to the customer. In this model, the scrap cost and the rework cost were assumed to be the same. However, the scrap cost and rework cost may not be same in many real manufacturing cases. For example, in a metal-cutting or machining process, if the diameter of a shaft is greater than the upper specification limit (USL), the part may be reworkable. On the other hand, if the diameter of a shaft is less than the lower specification limit (LSL), the part may no longer be usable and has to be scrapped. In addition to the above asymmetric losses, tolerance asymmetry is common in many manufacturing processes. If the deviation from the ideal target of a quality characteristic is more costly in one direction than in the opposite direction, an asymmetric tolerance design should be considered. Maghsoodloo and Li (2000) considered both linear and quadratic loss functions in determining the optimal value of a process mean that minimizes the expected losses for an asymmetric tolerance design. However, the revised loss functions were not studied in their models. Feng and Kapur (2006) proposed models that use asymmetric quality loss functions in both quadratic and piecewise linear cases for determining the optimal process mean and specifications for inspection. However, only the expected scrap cost per shipped unit incurred by the producer was considered in their expected total cost models.

The objective of this study was to develop a new model without the limitation that the scrap cost and the rework cost have to be the same in determining the economic specification limits. The expected total cost includes the scrap cost, the rework cost, the inspection cost and the loss due to variation. Considering that the quality losses above the upper or lower specification limits may not be equal, Kapur's (1988) economic tolerance design model is revised as

$$
T C=L *+q_{1} \times S C+q_{2} \times R C+I C
$$

where $R C$ is the rework cost per unit, $L *$ is the expected loss per unit product shipped to the customer using the above-mentioned loss functions and $q_{1}$ and $q_{2}$ denote the probabilities of scrap and rework, respectively.

The article is structured as follows. First, the expected total losses were estimated using three different loss functions: a revised Taguchi quadratic loss function, an inverted normal loss function (INLF) and a revised inverted normal loss function (RINLF). Then, the best loss function is determined in the economic tolerance design and the relationships between process capability 
indices and expected loss per unit under normal distribution are derived. Finally, a comparative study of the economic tolerance design is conducted using a realistic example of the solder paste stencil printing process.

\section{Estimation of the total expected loss}

Assuming the quality characteristic $Y$ follows a normal distribution, i.e. $Y \sim N\left(\mu, \sigma^{2}\right)$, the expected loss per unit product can be derived for the revised Taguchi quadratic loss function, INIF and RINLF. The revised Taguchi quadratic loss function can be written as

$$
L_{Q}(y)= \begin{cases}k_{1}(y-T)^{2} & \text { if } y<T \\ k_{2}(y-T)^{2} & \text { if } y \geq T\end{cases}
$$

where $k_{1}$ and $k_{2}$ represent the coefficients of two different quality losses and $T$ is the target value. The INLF loss function can be written as

$$
L_{I N L F}(y)= \begin{cases}K_{1}\left\{1-\exp \left[-\frac{(y-T)^{2}}{2 \sigma_{L 1}^{2}}\right]\right\} & \text { if } y<T \\ K_{2}\left\{1-\exp \left[-\frac{(y-T)^{2}}{2 \sigma_{L 2}^{2}}\right]\right\} & \text { if } y \geq T\end{cases}
$$

and the RINLF loss function can be written as

$$
L_{R I N L F}(y)= \begin{cases}K_{1}\left\{1-\exp \left(-\frac{(y-L)^{2}}{2 \sigma_{L_{1}}^{2}}\right)\right\} & y<L \\ 0 & L \leq y \leq U \\ K_{2}\left\{1-\exp \left(-\frac{(y-U)^{2}}{2 \sigma_{L_{2}}^{2}}\right)\right\} & y>U\end{cases}
$$

where $U$ is the upper limit for the acceptable range of a quality characteristic and $L$ is the lower limit for the acceptable range of a quality characteristic since quality loss will not incur when the quality characteristic falls within the neighbourhood of a target value from the customer's point of view and $\sigma_{L_{1}}^{2}$ and $\sigma_{L_{2}}^{2}$ are the parameters for controlling the shape of a function depending on the realistic loss. Given the above loss functions, the formulae of the total expected losses per unit product under two types of quality characteristics, i.e. the 'nominal the best' (bilateral specification) and the 'smaller the better' (unilateral specification), are derived. When the quality characteristics are the 'larger the better' (unilateral specification), the derivation of total expected losses per unit product is very similar to the case of the smaller the better case. Note that the asymmetric loss function proposed by Baker (1990) is used for the revised Taguchi loss function. 


\subsection{Estimation of the expected losses for the nominal the best case}

\subsubsection{Expected loss estimation using the revised Taguchi loss function}

In case 1 no inspection is performed. The total expected losses per unit product is the expected loss per unit product shipped to the customer, which can be written as

$$
\begin{aligned}
L_{Q}= & E\left[L_{Q}(y)\right]=\int_{-\infty}^{\infty} L_{Q}(y) \times f(y) d y \\
= & \int_{-\infty}^{T} k_{1}(y-T)^{2} \times \frac{1}{\sqrt{2 \pi} \sigma} \exp \left[-\frac{(y-\mu)^{2}}{2 \sigma^{2}}\right] d y \\
& +\int_{T}^{\infty} k_{2}(y-T)^{2} \times \frac{1}{\sqrt{2 \pi} \sigma} \exp \left[-\frac{(y-\mu)^{2}}{2 \sigma^{2}}\right] d y \\
= & k_{1}\left[\sigma^{2}+\{\mu-T\}^{2}\right]+\left(k_{2}-k_{1}\right)\left\{\sigma(\mu-T) \phi\left(\frac{T-\mu}{\sigma}\right)\right. \\
& \left.+\left[(\mu-T)^{2}+\sigma^{2}\right]\left[1-\Phi\left(\frac{T-\mu}{\sigma}\right)\right]\right\}
\end{aligned}
$$

In case 2 a $100 \%$ inspection is performed. The expected loss per unit product shipped to the customer can be written as

$$
\begin{aligned}
L_{Q}^{*}= & E\left[L_{Q}(y)\right]=\int_{\mu-\eta_{1} \sigma}^{\mu+\eta_{2} \sigma} L_{Q}(y) \times f_{T}(y) d y \\
= & \int_{\mu-\eta_{1} \sigma}^{T} k_{1}(y-T)^{2} \times \frac{1}{q} \times \frac{1}{\sqrt{2 \pi} \sigma} \exp \left[-\frac{(y-\mu)^{2}}{2 \sigma^{2}}\right] d y \\
& +\int_{T}^{\mu+\eta_{2} \sigma} k_{2}(y-T)^{2} \frac{1}{q} \times \frac{1}{\sqrt{2 \pi} \sigma} \exp \left[-\frac{(y-\mu)^{2}}{2 \sigma^{2}}\right] d y \\
= & k_{1}\left[V\left(Y_{T}\right)+\left\{E\left(Y_{T}\right)-T\right\}^{2}\right]+\left(\frac{k_{2}-k_{1}}{q}\right)\left\{\left[(\mu-T)^{2}+\sigma^{2}\right]\left[\Phi\left(\eta_{2}\right)-\Phi\left(\frac{T-\mu}{\sigma}\right)\right]\right. \\
& \left.+\sigma(\mu-T) \phi\left(\frac{T-\mu}{\sigma}\right)-2 \sigma\left[(\mu-T)+\frac{\eta_{2}}{2}\right] \phi\left(\eta_{2}\right)\right\}
\end{aligned}
$$

where

$$
\begin{aligned}
E\left[Y_{T}\right] & =\mu+\frac{\sigma}{q}\left[\phi\left(\eta_{1}\right)-\phi\left(\eta_{2}\right)\right] \text { and } \\
V\left[Y_{T}\right] & =\sigma^{2}\left\{1-\frac{\eta_{1}}{q} \phi\left(\eta_{1}\right)-\frac{\eta_{2}}{q} \phi\left(\eta_{2}\right)-\left[\frac{\phi\left(\eta_{1}\right)-\phi\left(\eta_{2}\right)}{q}\right]^{2}\right\} \\
q 1 & =1-\Phi\left(\eta_{1}\right) \quad q 2=1-\Phi\left(\eta_{2}\right) \quad q=\Phi\left(\eta_{1}\right)+\Phi\left(\eta_{2}\right)-1
\end{aligned}
$$

where $\phi(\cdot)$ and $\Phi(\cdot)$ denote the standard normal probability density function and the cumulative distribution, respectively. Note that $(\mu-T) / \sigma \leq \eta_{1} \leq \mu / \sigma$ and $\eta_{2} \geq(T-\mu) / \sigma$. 


\subsubsection{Expected loss estimation using the INLF}

In case 1 no inspection is performed. The total expected losses per unit product can be written as

$$
\begin{aligned}
L_{I N L F}= & E\left[L_{I N L F}(y)\right]=\int_{-\infty}^{\infty} L_{I N L F}(y) \times f(y) d y \\
= & \int_{-\infty}^{T} K_{1}\left\{1-\exp \left[-\frac{(y-T)^{2}}{2 \sigma_{L 1}^{2}}\right]\right\} \times f(y) d y \\
& +\int_{T}^{\infty} K_{2}\left\{1-\exp \left[-\frac{(y-T)^{2}}{2 \sigma_{L 2}^{2}}\right]\right\} \times f(y) d y \\
= & \left.\left.K_{1}\left\{\Phi\left(\frac{T-\mu}{\sigma}\right)-\frac{\sigma_{L 1}}{\sqrt{\sigma^{2}+\sigma_{L 1}^{2}}} \times \exp \left[-\frac{(\mu-T)^{2}}{2\left(\sigma^{2}+\sigma_{L 1}^{2}\right)}\right] \times \Phi\right] \frac{\sigma_{L 1}(T-\mu)}{\sigma^{2}+\sigma_{L 1}^{2}}\right)\right\} \\
& +K_{2}\left\{1-\Phi\left(\frac{T-\mu}{\sigma}\right)-\frac{\sigma_{L 2}}{\sqrt{\sigma^{2}+\sigma_{L 2}^{2}}} \times \exp \left[-\frac{(\mu-T)^{2}}{2\left(\sigma^{2}+\sigma_{L 2}^{2}\right)}\right]\right. \\
& \left.\times\left[1-\Phi\left(\frac{\sigma_{L 2}(T-\mu)}{\sigma \sqrt{\sigma^{2}+\sigma_{L 2}^{2}}}\right)\right]\right\}
\end{aligned}
$$

In case 2 a $100 \%$ inspection is performed. The expected loss per unit product shipped to the customer can be written as

$$
\begin{aligned}
L_{I N L F}^{*}= & E\left[L_{I N L F}(y)\right]=\int_{\mu-\eta_{1} \sigma}^{\mu+\eta_{2} \sigma} L_{I N L F}(y) \times f_{T}(y) d y \\
= & \int_{\mu-\eta_{1} \sigma}^{T} K_{1}\left\{1-\exp \left[-\frac{(y-T)^{2}}{2 \sigma_{L 1}^{2}}\right]\right\} \times f_{T}(y) d y \\
& +\int_{T}^{\mu+\eta_{2} \sigma} K_{2}\left\{1-\exp \left[-\frac{(y-T)^{2}}{2 \sigma_{L 1}^{2}}\right]\right\} \times f_{T}(y) d y \\
= & \frac{K_{1}}{q}\left\{\Phi\left(\frac{T-\mu}{\sigma}\right)+\Phi\left(\eta_{1}\right)-1-\frac{\sigma_{L 1}}{\sqrt{\sigma^{2}+\sigma_{L 1}^{2}} \times\left\{\exp \left[-\frac{(\mu-T)^{2}}{2\left(\sigma^{2}+\sigma_{L 1}^{2}\right)}\right]\right\}}\right. \\
& \left.\left.\times \Phi\left(\frac{\sigma_{L 1}(T-\mu)}{\sigma \sqrt{\sigma^{2}+\sigma_{L 1}^{2}}}\right)-\Phi\left(\frac{\sigma(\mu-T)-\eta_{1}\left(\sigma^{2}+\sigma_{L 1}^{2}\right)}{\sigma_{L 1} \sqrt{\sigma^{2}+\sigma_{L 1}^{2}}}\right)\right]\right\} \\
& +\frac{K_{2}}{q}\left\{\Phi\left(\eta_{2}\right)-\Phi\left(\frac{T-\mu}{\sigma}\right)-\frac{\sigma_{L 2}}{\sqrt{\sigma^{2}+\sigma_{L 2}^{2}} \times\left\{\exp \left[-\frac{(\mu-T)^{2}}{2\left(\sigma^{2}+\sigma_{L 2}^{2}\right)}\right]\right\}}\right. \\
& \times\left[\Phi\left(\frac{\sigma(\mu-T)+\eta_{2}\left(\sigma^{2}+\sigma_{L 2}^{2}\right)}{\sigma_{L 2} \sqrt{\sigma^{2}+\sigma_{L 2}^{2}}}\right)\right. \\
& {\left.\left.\left[\frac{\sigma_{L 2}(T-\mu)}{\sigma \sqrt{\sigma^{2}+\sigma_{L 2}^{2}}}\right)\right]\right\} }
\end{aligned}
$$




\subsubsection{Expected loss estimation using the RINLF}

In case 1 no inspection is performed. The total expected losses per unit product can be written as

$$
\begin{aligned}
L_{R I N L F}= & E\left[L_{R I N L F}(y)\right]=\int_{-\infty}^{\infty} L_{R I N L F}(y) \times f(y) d y \\
= & \int_{-\infty}^{L} K_{1}\left\{1-\exp \left[-\frac{(y-L)^{2}}{2 \sigma_{L 1}^{2}}\right]\right\} \times f(y) d y \\
& +\int_{U}^{\infty} K_{2}\left\{1-\exp \left[-\frac{(y-U)^{2}}{2 \sigma_{L 2}^{2}}\right]\right\} \times f(y) d y \\
= & \left.\left.K_{1}\left\{\Phi\left(\frac{L-\mu}{\sigma}\right)-\frac{\sigma_{L 1}}{\sqrt{\sigma^{2}+\sigma_{L 1}^{2}} \times \exp \left[-\frac{(\mu-L)^{2}}{2\left(\sigma^{2}+\sigma_{L 1}^{2}\right)}\right] \times \Phi}\right] \frac{\sigma_{L 1}(L-\mu)}{\sigma^{2}+\sigma_{L 1}^{2}}\right)\right\} \\
& +K_{2}\left\{1-\Phi\left(\frac{U-\mu}{\sigma}\right)-\frac{\sigma_{L 2}}{\sqrt{\sigma^{2}+\sigma_{L 2}^{2}}} \times \exp \left[-\frac{(\mu-U)^{2}}{2\left(\sigma^{2}+\sigma_{L 2}^{2}\right)}\right]\right. \\
& \times\left[1-\Phi\left(\frac{\sigma_{L 2}(U-\mu)}{\left.\left.\left.\sigma_{\sqrt{\sigma^{2}+\sigma_{L 2}^{2}}}\right)\right]\right\}}\right.\right.
\end{aligned}
$$

In case 2 a $100 \%$ inspection is performed. The expected loss per unit product shipped to the customer can be written as

$$
\begin{aligned}
& L_{R I N L F}^{*}=E\left[L_{R I N L F}(y)\right]=\int_{\mu-\eta_{1} \sigma}^{\mu+\eta_{2} \sigma} L_{R I N L F}(y) \times f_{T}(y) d y \\
& =\int_{\mu-\eta_{1} \sigma}^{L} K_{1}\left\{1-\exp \left[-\frac{(y-L)^{2}}{2 \sigma_{L 1}^{2}}\right]\right\} \times f_{T}(y) d y \\
& +\int_{U}^{\mu+\eta_{2} \sigma} K_{2}\left\{1-\exp \left[-\frac{(y-U)^{2}}{2 \sigma_{L 1}^{2}}\right]\right\} \times f_{T}(y) d y \\
& =\frac{K_{1}}{q}\left\{\Phi\left(\frac{L-\mu}{\sigma}\right)+\Phi\left(\eta_{1}\right)-1-\frac{\sigma_{L 1}}{\sqrt{\sigma^{2}+\sigma_{L 1}^{2}}} \times\left\{\exp \left[-\frac{(\mu-L)^{2}}{2\left(\sigma^{2}+\sigma_{L 1}^{2}\right)}\right]\right\}\right. \\
& \left.\times\left[\Phi\left(\frac{\sigma_{L 1}(L-\mu)}{\sigma \sqrt{\sigma^{2}+\sigma_{L 1}^{2}}}\right)-\Phi\left(\frac{\sigma(\mu-L)-\eta_{1}\left(\sigma^{2}+\sigma_{L 1}^{2}\right)}{\sigma_{L 1} \sqrt{\sigma^{2}+\sigma_{L 1}^{2}}}\right)\right]\right\} \\
& +\frac{K_{2}}{q}\left\{\Phi\left(\eta_{2}\right)-\Phi\left(\frac{U-\mu}{\sigma}\right)-\frac{\sigma_{L 2}}{\sqrt{\sigma^{2}+\sigma_{L 2}^{2}}} \times\left\{\exp \left[-\frac{(\mu-U)^{2}}{2\left(\sigma^{2}+\sigma_{L 2}^{2}\right)}\right]\right\}\right. \\
& \left.\times\left[\Phi\left(\frac{\sigma(\mu-U)+\eta_{2}\left(\sigma^{2}+\sigma_{L 2}^{2}\right)}{\sigma_{L 2} \sqrt{\sigma^{2}+\sigma_{L 2}^{2}}}\right)-\Phi\left(\frac{\sigma_{L 2}(U-\mu)}{\sigma \sqrt{\sigma^{2}+\sigma_{L 2}^{2}}}\right)\right]\right\}
\end{aligned}
$$

where $\eta_{1} \geq(\mu-L) / \sigma$ and $\eta_{2} \geq(U-\mu) / \sigma$. 


\subsection{Estimation of the expected losses for the smaller the better case}

When the quality characteristics are the smaller the better (unilateral specification), assume the economic $U S L=\mu+\eta_{1} \sigma$ and the product will be reworked or scrapped if $Y$ exceeds the USL.

\subsubsection{Expected loss estimation using the revised Taguchi loss function}

In case 1 no inspection is performed. The total expected losses per unit product is the expected loss per unit product shipped to the customer, which can be written as

$$
L_{Q}=E\left[L_{Q}(y)\right]=\int_{0}^{\infty} L_{Q}(y) \times f(y) d y=\int_{0}^{\infty} k y^{2} \times f(y) d y=k\left[\sigma^{2}+\mu^{2}\right]
$$

In case 2 a $100 \%$ inspection is performed. The expected loss per unit product shipped to the customer can be written as

$$
\begin{aligned}
L_{Q}^{*} & =E\left[L_{Q}(y)\right]=\int_{0}^{\mu+\eta \sigma} L_{Q}(y) \times f_{T}(y) d y=\int_{0}^{\mu+\eta \sigma} k y^{2} \times f_{T}(y) d y \\
& =k\left\{V\left(Y_{T}\right)+\left[E\left(Y_{T}\right)\right]^{2}\right\}
\end{aligned}
$$

where

$$
E\left[Y_{T}\right]=\mu-\sigma / q[\phi(\eta) / \Phi(\eta)] V\left[Y_{T}\right]=\sigma^{2}\left\{1-\eta[\phi(\eta) / \Phi(\eta)]-[\phi(\eta) / \Phi(\eta)]^{2}\right\}
$$

\subsubsection{Expected loss estimation using the INLF}

In case 1 no inspection is performed. The total expected losses per unit product can be written as

$$
\begin{aligned}
L_{I N L F} & =E\left[L_{I N L F}(y)\right]=\int_{0}^{\infty} L_{I N L F}(y) \times f(y) d y=\int_{0}^{\infty} K\left\{1-\exp \left(-\frac{y^{2}}{2 \sigma_{L}^{2}}\right)\right\} \times f(y) d y \\
& =K \times\left[\Phi\left(\frac{\mu}{\sigma}\right)-\frac{\sigma_{L}}{\sqrt{\sigma^{2}+\sigma_{L}^{2}}} \times \exp \left(-\frac{\mu^{2}}{2\left(\sigma^{2}+\sigma_{L}^{2}\right)}\right) \times \Phi\left(\frac{\mu \times \sigma_{L}}{\sigma \sqrt{\sigma^{2}+\sigma_{L}^{2}}}\right)\right]
\end{aligned}
$$

In case 2 a $100 \%$ inspection is performed. The expected loss per unit product shipped to the customer can be written as

$$
\begin{aligned}
L_{I N L F}^{*}= & E\left[L_{I N L F}(y)\right]=\int_{0}^{\mu+\eta \sigma} L_{I N L F}(y) \times f_{T}(y) d y \\
= & \int_{0}^{\mu+\eta \sigma} K\left\{1-\exp \left[-\frac{y^{2}}{2 \sigma_{L}^{2}}\right]\right\} \times f_{T}(y) d y \\
= & \frac{K}{q}\left\{\Phi(\eta)-\Phi\left(\frac{-\mu}{\sigma}\right)-\frac{\sigma_{L}}{\sqrt{\sigma^{2}+\sigma_{L}^{2}}} \times\left\{\exp \left[-\frac{\mu^{2}}{2\left(\sigma^{2}+\sigma_{L}^{2}\right)}\right]\right\}\right. \\
& \left.\times\left[\Phi\left(\frac{\sigma(\mu)+\eta\left(\sigma^{2}+\sigma_{L}^{2}\right)}{\sigma_{L} \sqrt{\sigma^{2}+\sigma_{L}^{2}}}\right)-\Phi\left(\frac{\sigma_{L}(-\mu)}{\sigma \sqrt{\sigma^{2}+\sigma_{L}^{2}}}\right)\right]\right\}
\end{aligned}
$$




\subsubsection{Expected loss estimation using the RINLF}

In case 1 no inspection is performed. The total expected losses per unit product can be written as

$$
\begin{aligned}
L_{R I N L F}= & E\left[L_{R I N L F}(y)\right]=\int_{0}^{\infty} L_{R I N L F}(y) \times f(y) d y \\
= & \int_{U}^{\infty} K\left\{1-\exp \left[-\frac{(y-U)^{2}}{2 \sigma_{L}^{2}}\right]\right\} \times f(y) d y \\
= & K\left\{1-\Phi\left(\frac{U-\mu}{\sigma}\right)-\frac{\sigma_{L}}{\sqrt{\sigma^{2}+\sigma_{L}^{2}}} \times \exp \left(-\frac{(\mu-U)^{2}}{2\left(\sigma^{2}+\sigma_{L}^{2}\right)}\right)\right. \\
& \left.\times\left[1-\Phi\left(\frac{\sigma_{L}(U-\mu)}{\sigma \sqrt{\sigma^{2}+\sigma_{L}^{2}}}\right)\right]\right\}
\end{aligned}
$$

In case 2 a $100 \%$ inspection is performed. The expected loss per unit product shipped to the customer can be written as

$$
\begin{aligned}
L_{R I N L F}^{*}= & E\left[L_{R I N L F}(y)\right]=\int_{U}^{\mu+\eta \sigma} L_{R I N L F}(y) \times f_{T}(y) d y \\
= & \int_{U}^{\mu+\eta \sigma} K\left\{1-\exp \left[-\frac{(y-U)^{2}}{2 \sigma_{L}^{2}}\right]\right\} \times f_{T}(y) d y \\
= & \frac{K}{q}\left\{\Phi(\eta)-\Phi\left(\frac{U-\mu}{\sigma}\right)-\frac{\sigma_{L}}{\sqrt{\sigma^{2}+\sigma_{L}^{2}}} \times\left\{\exp \left[-\frac{(\mu-U)^{2}}{2\left(\sigma^{2}+\sigma_{L}^{2}\right)}\right]\right\}\right. \\
& \left.\times\left[\Phi\left(\frac{\sigma(\mu-U)+\eta\left(\sigma^{2}+\sigma_{L}^{2}\right)}{\sigma_{L} \sqrt{\sigma^{2}+\sigma_{L}^{2}}}\right)-\Phi\left(\frac{\sigma_{L}(U-\mu)}{\sigma \sqrt{\sigma^{2}+\sigma_{L}^{2}}}\right)\right]\right\}
\end{aligned}
$$

\section{Relationship between loss functions and process capability indices}

To answer which loss function is appropriate in determining the economic specification limits, it is necessary to explore the relationship between loss function and process capability indices. Assuming that a quality characteristics $Y$ follows a normal distribution, the process capability indices for a bilateral specification are

$$
\begin{aligned}
C_{p} & =\frac{U S L-L S L}{6 \sigma}=\frac{\Delta}{3 \sigma} \\
C_{p k} & =\min \left\{C_{p l}, C_{p u}\right\}=\min \left\{\frac{\mu-L S L}{3 \sigma}, \frac{U S L-\mu}{3 \sigma}\right\}=\frac{\Delta-|\mu-M|}{3 \sigma} \\
C_{p m} & =\frac{U S L-L S L}{6\left\{E(Y-T)^{2}\right\}^{1 / 2}}=\frac{\Delta}{3 \sqrt{\sigma^{2}+(\mu-T)^{2}}}
\end{aligned}
$$

where $M=U S L+L S L / 2$ is the centre of a bilateral specification and $\Delta=U S L-L S L / 2$ denotes half of the specification width. The relationships between loss functions and process capability indices for both symmetric and asymmetric tolerances are derived as below. 


\subsection{Relationship between loss functions and process capability indices for symmetric tolerance}

Assuming that the quality characteristic of a symmetric tolerance $(T=M)$, as shown in Figure 1, follows a normal distribution and its process capability indices $C_{p}$ and $C_{p k}$ are known, one can obtain $|\mu-M|=3 \sigma\left(C_{p}-C_{p k}\right)$. Thus, the economic lower and upper specifications are

$$
\begin{aligned}
L S L & =\mu-\eta_{1} \sigma \Longrightarrow \eta_{1}=[\Delta-(T-\mu)] / \sigma=\left[3 \sigma C_{p}-(T-\mu)\right] / \sigma \\
U S L & =\mu+\eta_{2} \sigma \Longrightarrow \eta_{2}=\left[3 \sigma C_{p}+(T-\mu)\right] / \sigma
\end{aligned}
$$

When the process average $\mu$ is equal to the target value $T$, then $C_{p}=C_{p k}$ and $\eta_{1}=\eta_{2}=\eta=3 C_{p}$. Note that only the asymmetric loss $(S C \neq R C)$ is plotted in Figure 1 since the symmetric loss $(S C=R C)$ can be considered as a special case of asymmetric loss. By utilizing $C_{p}$, one can derive the following relationships between three different loss functions and process capability indices.

\subsubsection{The relationship between the revised Taguchi's loss function and process capability indices}

Assume the quality losses exceed the upper or lower specification limits which are not equal and $k_{1}$ and $k_{2}$ represent the coefficients of two different quality losses, namely

$$
\begin{aligned}
& k_{1}=K_{1} / \Delta^{2}=K_{1} /\left(3 \sigma C_{p}\right)^{2} \\
& k_{2}=K_{2} / \Delta^{2}=K_{2} /\left(3 \sigma C_{p}\right)^{2}
\end{aligned}
$$

where $K_{1}$ denotes the maximum loss including both producer and consumer costs if the quality characteristic deviates from the target and exceeds the LSL and $K_{2}$ denotes the maximum loss including both producer and consumer costs if the characteristic deviates from the target and exceeds the USL. The expected loss per unit product shipped to the customer can be written as

$$
L_{Q}^{*}=\left(K_{1}+K_{2}\right)\left\{\frac{2 \Phi\left(3 C_{p}\right)-6 \phi\left(3 C_{p}\right) C_{p}-1}{18\left[2 \Phi\left(3 C_{p}\right)-1\right] C_{p}^{2}}\right\}
$$

Considering the scrap, rework and inspection costs, the total expected loss per unit product is

$$
T C=L_{Q}^{*}+\left[1-\Phi\left(3 C_{p}\right)\right] \times(S C+R C)+I C
$$

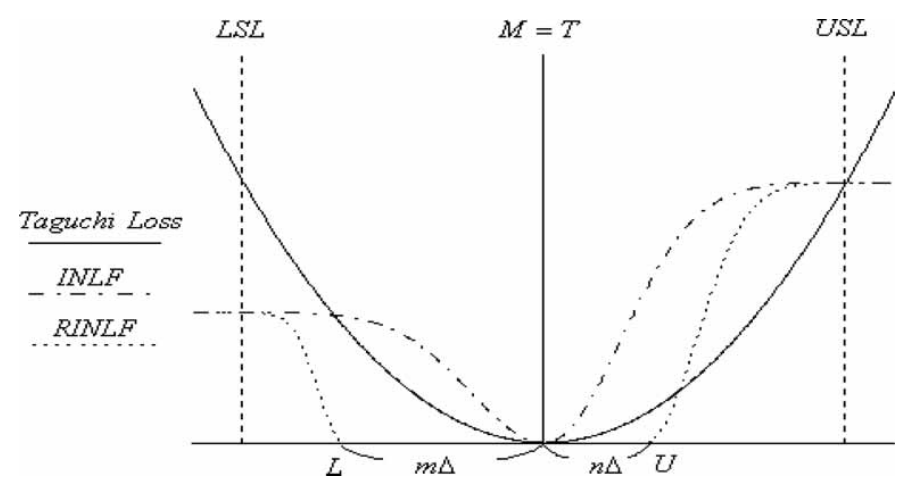

Figure 1. Comparison of three loss functions for symmetric tolerance $(T=M)$. 


\subsubsection{The relationship between the INLF and process capability indices}

At the specification limits, the loss reaches its maximum value. Therefore, the parameters are set according to the rule proposed by Spiring (1993): $\sigma_{L 1}=\sigma_{L 2}=\Delta / 4=[(3 \sigma) 4] C_{p}$. The expected loss per unit product shipped to the customer can be written as

$$
L_{I N L F}^{*}=\frac{\left(K_{1}+K_{2}\right)}{2 \Phi\left(3 C_{p}\right)-1} \times\left\{\Phi\left(3 C_{p}\right)-\frac{1}{2}-\frac{3 C_{p}}{\sqrt{16+9 C_{p}^{2}}}\left[\Phi\left(\sqrt{16+9 C_{p}^{2}}\right)-\frac{1}{2}\right]\right\}
$$

The total expected losses per unit product is $T C=L_{I N L F}^{*}+\left[1-\Phi\left(3 C_{p}\right)\right] \times(S C+R C)+I C$.

\subsubsection{The relationship between RINLF and process capability indices}

The parameters are set according to the rule proposed by Spiring (1993):

$$
\begin{gathered}
\sigma_{L 1}=\frac{L-L S L}{4}=\frac{3 \sigma(1-m) C_{p}}{4} \\
\sigma_{L 2}=\frac{U S L-U}{4}=\frac{3 \sigma(1-n) C_{p}}{4} .
\end{gathered}
$$

The expected loss per unit product shipped to the customer can be written as

$$
\begin{aligned}
L_{R I N L F}^{*}= & \frac{K_{1}}{2 \Phi\left(3 C_{p}\right)-1}\left\{\Phi\left(-3 m C_{p}\right)+\Phi\left(3 C_{p}\right)-1\right. \\
& -\left\{\exp \left[-\frac{72 m^{2} C_{p}^{2}}{16+9(1-m)^{2} C_{p}^{2}}\right]\right\} \times \frac{3(1-m) C_{p}}{\sqrt{16+9(1-m)^{2} C_{p}^{2}}} \\
& \left.\left.\times \Phi\left(-\frac{9 m(1-m) C_{p}^{2}}{\sqrt{16+9(1-m)^{2} C_{p}^{2}}}\right)-\Phi\left(\frac{-16-9(1-m) C_{p}^{2}}{\sqrt{16+9(1-m)^{2} C_{p}^{2}}}\right)\right]\right\} \\
& +\frac{K_{2}}{2 \Phi\left(3 C_{p}\right)-1} \times\left\{\left(3 C_{p}\right)-\Phi\left(3 n C_{p}\right)-\left\{\exp \left[-\frac{72 n^{2} C_{p}^{2}}{16+9(1-n)^{2} C_{p}^{2}}\right]\right\}\right. \\
& \left.\times \frac{3(1-n) C_{p}}{\sqrt{16+9(1-n)^{2} C_{p}^{2}}}\left[\Phi\left(-\frac{-16-9(1-n) C_{p}^{2}}{\sqrt{16+9(1-n)^{2} C_{p}^{2}}}\right)-\Phi\left(\frac{9 n(1-n) C_{p}^{2}}{\sqrt{16+9(1-n)^{2} C_{p}^{2}}}\right)\right]\right\}
\end{aligned}
$$

where $m=T-L / \Delta$ denotes the difference between the target and the lower limit for the acceptable range, in which no quality loss will be incurred, divided by half of the specification width: here $n=U-T / \Delta$ denotes the difference between the target and the upper limit for the acceptable range, divided by half of the specification width. According to Equation (2), the total expected loss per unit product is $T C=L_{R I N L F}^{*}+\left[1-\Phi\left(3 C_{p}\right)\right] \times(S C+R C)+I C$. 
When a process average $\mu$ is greater or less than the target value $T$, the derivation of the relationship between the process capability indices and loss functions is similar to the case of the process average $\mu=T$, as described above.

Based on the relationship between various loss functions and process capability indices for symmetric tolerance, the comparison of expected loss estimation using three loss functions is shown in Table 1. It shows that, given the maximum loss of scrap $K_{1}$ and the maximum loss of rework $K_{2}$, then the three expected losses per unit can be estimated with respect to different $C_{p}$ indices and their corresponding defect rates are also indicated accordingly.

\subsection{The relationship between loss function and process capability indices for asymmetric tolerance}

When the quality characteristic of an asymmetric tolerance $(T \neq M)$, as shown in Figure 2, follows a normal distribution and its process capability indices $C_{p}$ and $C_{p m}$ are known, then let $d=T-M / \Delta$ denote the difference between the target and the centre of specification divided by half of the specification width, where $|d| \leq 1$. Then $C_{p}=\Delta / 3 \sigma \Rightarrow \Delta=3 \sigma C_{p}$ and $C_{p m}=$ $\Delta /\left(3 \sqrt{\sigma^{2}+(\mu-T)^{2}}\right)=\left(\sigma \times C_{p}\right) / \sqrt{\sigma^{2}+(\mu-T)^{2}}$. Hence,

$$
\begin{aligned}
|\mu-T| & =\left(\sigma / C_{p m}\right) \sqrt{C_{p}^{2}-C_{p m}^{2}} \\
\mathrm{LSL} & =\mu-\eta_{1} \sigma \Longrightarrow \eta_{1}=[\Delta(1+d)-(T-\mu)] / \sigma=\left[3 \sigma(1+d) C_{p}-(T-\mu)\right] / \sigma \\
\mathrm{USL} & =\mu+\eta_{2} \sigma \Longrightarrow \eta_{2}=\left[3 \sigma(1-d) C_{p}+(T-\mu)\right] / \sigma .
\end{aligned}
$$

\begin{tabular}{|c|c|c|c|c|}
\hline \multirow[b]{2}{*}{$C_{p}$} & \multirow{2}{*}{$\begin{array}{l}\text { Defect rate } \\
\text { (p.p.m) }\end{array}$} & \multicolumn{3}{|c|}{ Expected Unit Loss } \\
\hline & & Revised Taguchi & INLF & RINLF \\
\hline 0.10 & 764177.1556 & $5.541 *\left(\mathrm{~K}_{1}+\mathrm{K}_{2}\right)$ & $0.341 *\left(\mathrm{~K}_{1}+\mathrm{K}_{2}\right)$ & $0.169 *\left(\mathrm{~K}_{1}+\mathrm{K}_{2}\right)$ \\
\hline 0.20 & 548506.2355 & $1.382 *\left(\mathrm{~K}_{1}+\mathrm{K}_{2}\right)$ & $0.336 *\left(\mathrm{~K}_{1}+\mathrm{K}_{2}\right)$ & $0.161 *\left(\mathrm{~K}_{1}+\mathrm{K}_{2}\right)$ \\
\hline 0.30 & 368120.2507 & $0.612 *\left(\mathrm{~K}_{1}+\mathrm{K}_{2}\right)$ & $0.326 *\left(\mathrm{~K}_{1}+\mathrm{K}_{2}\right)$ & $0.148 *\left(\mathrm{~K}_{1}+\mathrm{K}_{2}\right)$ \\
\hline 0.33 & 322174.1190 & $0.506 *\left(\mathrm{~K}_{1}+\mathrm{K}_{2}\right)$ & $0.323 *\left(\mathrm{~K}_{1}+\mathrm{K}_{2}\right)$ & $0.144 *\left(\mathrm{~K}_{1}+\mathrm{K}_{2}\right)$ \\
\hline 0.40 & 230139.3404 & $0.344 *\left(\mathrm{~K}_{1}+\mathrm{K}_{2}\right)$ & $0.313 *\left(\mathrm{~K}_{1}+\mathrm{K}_{2}\right)$ & $0.132 *\left(\mathrm{~K}_{1}+\mathrm{K}_{2}\right)$ \\
\hline 0.50 & 133614.4025 & $0.219 *\left(\mathrm{~K}_{1}+\mathrm{K}_{2}\right)$ & $0.297 *\left(\mathrm{~K}_{1}+\mathrm{K}_{2}\right)$ & $0.112 *\left(\mathrm{~K}_{1}+\mathrm{K}_{2}\right)$ \\
\hline 0.60 & 71860.6382 & $0.152 *\left(\mathrm{~K}_{1}+\mathrm{K}_{2}\right)$ & $0.279 *\left(\mathrm{~K}_{1}+\mathrm{K}_{2}\right)$ & $0.092 *\left(\mathrm{~K}_{1}+\mathrm{K}_{2}\right)$ \\
\hline 0.67 & 44431.1889 & $0.122 *\left(\mathrm{~K}_{1}+\mathrm{K}_{2}\right)$ & $0.265 *\left(\mathrm{~K}_{1}+\mathrm{K}_{2}\right)$ & $0.078 *\left(\mathrm{~K}_{1}+\mathrm{K}_{2}\right)$ \\
\hline 0.70 & 35728.8411 & $0.111 *\left(\mathrm{~K}_{1}+\mathrm{K}_{2}\right)$ & $0.259 *\left(\mathrm{~K}_{1}+\mathrm{K}_{2}\right)$ & $0.072 *\left(\mathrm{~K}_{1}+\mathrm{K}_{2}\right)$ \\
\hline 0.80 & 16395.0718 & $0.085 *\left(\mathrm{~K}_{1}+\mathrm{K}_{2}\right)$ & $0.239 *\left(\mathrm{~K}_{1}+\mathrm{K}_{2}\right)$ & $0.054 *\left(\mathrm{~K}_{1}+\mathrm{K}_{2}\right)$ \\
\hline 0.90 & 6933.9476 & $0.067 *\left(\mathrm{~K}_{1}+\mathrm{K}_{2}\right)$ & $0.218 *\left(\mathrm{~K}_{1}+\mathrm{K}_{2}\right)$ & $0.038 *\left(\mathrm{~K}_{1}+\mathrm{K}_{2}\right)$ \\
\hline 1.00 & 2699.7961 & $0.054 *\left(\mathrm{~K}_{1}+\mathrm{K}_{2}\right)$ & $0.199 *\left(\mathrm{~K}_{1}+\mathrm{K}_{2}\right)$ & $0.026 *\left(\mathrm{~K}_{1}+\mathrm{K}_{2}\right)$ \\
\hline 1.10 & 966.8483 & $0.045 *\left(\mathrm{~K}_{1}+\mathrm{K}_{2}\right)$ & $0.182 *\left(\mathrm{~K}_{1}+\mathrm{K}_{2}\right)$ & $0.018 *\left(\mathrm{~K}_{1}+\mathrm{K}_{2}\right)$ \\
\hline 1.20 & 318.2172 & $0.037 *\left(\mathrm{~K}_{1}+\mathrm{K}_{2}\right)$ & $0.165 *\left(\mathrm{~K}_{1}+\mathrm{K}_{2}\right)$ & $0.011 *\left(\mathrm{~K}_{1}+\mathrm{K}_{2}\right)$ \\
\hline 1.30 & 96.1927 & $0.032 *\left(\mathrm{~K}_{1}+\mathrm{K}_{2}\right)$ & $0.151 *\left(\mathrm{~K}_{1}+\mathrm{K}_{2}\right)$ & $0.007 *\left(\mathrm{~K}_{1}+\mathrm{K}_{2}\right)$ \\
\hline 1.33 & 66.0733 & $0.030 *\left(\mathrm{~K}_{1}+\mathrm{K}_{2}\right)$ & $0.147 *\left(\mathrm{~K}_{1}+\mathrm{K}_{2}\right)$ & $0.0061 *\left(\mathrm{~K}_{1}+\mathrm{K}_{2}\right)$ \\
\hline 1.40 & 26.6915 & $0.027 *\left(\mathrm{~K}_{1}+\mathrm{K}_{2}\right)$ & $0.138 *\left(\mathrm{~K}_{1}+\mathrm{K}_{2}\right)$ & $0.0043 *\left(\mathrm{~K}_{1}+\mathrm{K}_{2}\right)$ \\
\hline 1.50 & 6.7953 & $0.024 *\left(\mathrm{~K}_{1}+\mathrm{K}_{2}\right)$ & $0.126 *\left(\mathrm{~K}_{1}+\mathrm{K}_{2}\right)$ & $0.0033 *\left(\mathrm{~K}_{1}+\mathrm{K}_{2}\right)$ \\
\hline 1.60 & 1.5867 & $0.021 *\left(\mathrm{~K}_{1}+\mathrm{K}_{2}\right)$ & $0.116 *\left(\mathrm{~K}_{1}+\mathrm{K}_{2}\right)$ & $0.0015 *\left(\mathrm{~K}_{1}+\mathrm{K}_{2}\right)$ \\
\hline 1.67 & 0.5443 & $0.019 *\left(\mathrm{~K}_{1}+\mathrm{K}_{2}\right)$ & $0.109 *\left(\mathrm{~K}_{1}+\mathrm{K}_{2}\right)$ & $0.0010 *\left(\mathrm{~K}_{1}+\mathrm{K}_{2}\right)$ \\
\hline 1.70 & 0.3397 & $0.018 *\left(\mathrm{~K}_{1}+\mathrm{K}_{2}\right)$ & $0.107 *\left(\mathrm{~K}_{1}+\mathrm{K}_{2}\right)$ & $0.00085 *\left(\mathrm{~K}_{1}+\mathrm{K}_{2}\right)$ \\
\hline 1.80 & 0.0666 & $0.016 *\left(\mathrm{~K}_{1}+\mathrm{K}_{2}\right)$ & $0.098 *\left(\mathrm{~K}_{1}+\mathrm{K}_{2}\right)$ & $0.00047 *\left(\mathrm{~K}_{1}+\mathrm{K}_{2}\right)$ \\
\hline 1.90 & 0.0120 & $0.015 *\left(\mathrm{~K}_{1}+\mathrm{K}_{2}\right)$ & $0.091 *\left(\mathrm{~K}_{1}+\mathrm{K}_{2}\right)$ & $0.00026 *\left(\mathrm{~K}_{1}+\mathrm{K}_{2}\right)$ \\
\hline 2.00 & 0.0020 & $0.013 *\left(\mathrm{~K}_{1}+\mathrm{K}_{2}\right)$ & $0.084 *\left(\mathrm{~K}_{1}+\mathrm{K}_{2}\right)$ & $0.00014 *\left(\mathrm{~K}_{1}+\mathrm{K}_{2}\right)$ \\
\hline
\end{tabular}

Table 1. A comparison of three loss functions $(m=0.5, n=0.5)$ for different $C_{p}$ indices. 


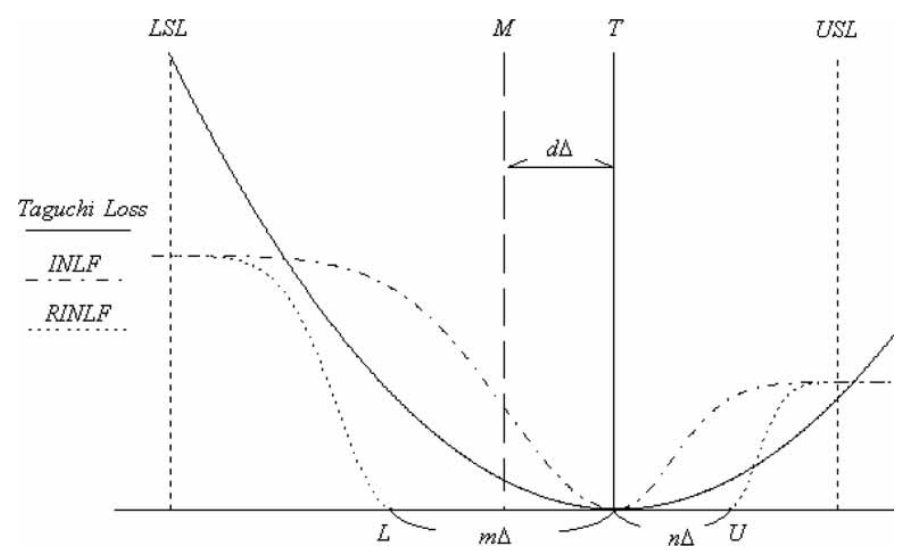

Figure 2. Comparison of three loss functions for asymmetric tolerance $(T \neq M)$.

By utilizing the relationship between the process capability indices $C_{p}$ and $C_{p m}$ and various loss functions, the expected loss per unit product can be obtained.

If a process average is $\mu=T$, then $\eta_{1}=3(1+d) C_{p}$ and $\eta_{2}=3(1-d) C_{p}$. Again, only the asymmetric loss $(S C \neq R C)$ is plotted in Figure 2 since the symmetric loss $(S C=R C)$ can be considered as a special case of asymmetric loss. By utilizing $C_{p}$, one can derive the following relationships between three different loss functions and process capability indices:

\subsubsection{The relationship between the revised Taguchi loss function and process capability indices}

Assuming the quality losses exceed the upper or lower specification limit and are not equal, let $k_{1}$ and $k_{2}$ represent the coefficients of two different quality losses, namely

$$
k_{1}=K_{1} /\left(\eta_{1} \sigma\right)^{2}=K_{1} /\left(9(1+d)^{2} \sigma^{2} C_{p}^{2}\right), k_{2}=K_{2} /\left(\eta_{2} \sigma\right)^{2}=K_{2} /\left(9(1-d)^{2} \sigma^{2} C_{p}^{2}\right)
$$

where $K_{1}$ denotes the maximum loss including both the producer and consumer costs if the quality characteristic deviates from the target and exceeds the LSL and $K_{2}$ denotes the maximum loss including both the producer and consumer costs if the characteristic deviates from the target and exceeds the USL. The expected loss per unit product shipped to the customer can be written as

$$
\begin{aligned}
L_{Q}^{*}= & K_{1}\left[\frac{2 \Phi\left[3(1+d) C_{p}\right]-6 \phi\left[3(1+d) C_{p}\right] C_{p}(1+d)-1}{18\left[\Phi\left[3(1+d) C_{p}\right]+\Phi\left[3(1-d) C_{p}\right]-1\right](1+d)^{2} C_{p}^{2}}\right] \\
& +K_{2}\left[\frac{2 \Phi\left[3(1-d) C_{p}\right]-6 \phi\left[3(1-d) C_{p}\right] C_{p}(1-d)-1}{18\left[\Phi\left[3(1+d) C_{p}\right]+\Phi\left[3(1-d) C_{p}\right]-1\right](1-d)^{2} C_{p}^{2}}\right]
\end{aligned}
$$

According to Equation (2) the total expected loss per unit product is

$$
T C=L_{Q}^{*}+\left[1-\Phi\left(3(1+d) C_{p}\right)\right] \times S C+\left[1-\Phi\left(3(1-d) C_{p}\right)\right] \times R C+I C
$$




\subsubsection{The relationship between the INLF and process capability indices}

Set the parameters according to the rule proposed by Spiring (1993):

$$
\sigma_{L 1}=\frac{T-L S L}{4}=\frac{3 \sigma(1+d) C_{p}}{4}
$$

and

$$
\sigma_{L 2}=\frac{U S L-T}{4}=\frac{3 \sigma(1-d) C_{p}}{4} .
$$

The expected loss per unit product shipped to the customer can be written as

$$
\begin{aligned}
L_{\mathrm{INLF}}^{*}= & \frac{K_{1}}{\Phi\left[3(1+d) C_{p}\right]+\Phi\left[3(1-d) C_{p}\right]-1}\left\{\Phi\left[3(1+d) C_{p}\right]\right. \\
& -\frac{1}{2}-\frac{3 C_{p}(1+d)}{\sqrt{16+9(1+d)^{2} C_{p}^{2}}}\left[\frac{1}{2}-\Phi\left(-\sqrt{\left.\left.\left.16+9(1+d)^{2} C_{p}^{2}\right)\right]\right\}}\right.\right. \\
& +\frac{K_{2}}{\Phi\left[3(1+d) C_{p}\right]+\Phi\left[3(1-d) C_{p}\right]-1}\left\{\Phi\left[3(1-d) C_{p}\right]\right. \\
& \left.-\frac{1}{2}-\frac{3 C_{p}(1-d)}{\sqrt{16+9(1-d)^{2} C_{p}^{2}}}\left[\Phi\left(\sqrt{16+9(1-d)^{2} C_{p}^{2}}\right)-\frac{1}{2}\right]\right\}
\end{aligned}
$$

According to Equation (2), the total expected loss per unit product is

$$
T C=L_{I N L F}^{*}+\left[1-\Phi\left(3(1+d) C_{p}\right)\right] \times S C+\left[1-\Phi\left(3(1-d) C_{p}\right)\right] \times R C+I C
$$

\subsubsection{The relationship between the RINLF and process capability indices}

Since the loss reaches its maximum value at the specification limits, the parameters are set according to the rule proposed by Spiring (1993) as

$$
\sigma_{L 1}=\frac{L-L S L}{4}=\frac{3 \sigma(1-m+d) C_{p}}{4} ; \quad \sigma_{L 2}=\frac{U S L-U}{4}=\frac{3 \sigma(1-n-d) C_{p}}{4} .
$$


The expected loss per unit product shipped to the customer can be written as

$$
\begin{aligned}
L_{R I N L F}^{*}= & \frac{K_{1}}{\Phi\left[3(1+d) C_{p}\right]+\Phi\left[3(1-d) C_{p}\right]-1}\left\{\Phi\left(-3 m C_{p}\right)+\Phi\left[3(1+d) C_{p}\right]-1\right. \\
& -\left\{\exp \left[-\frac{72 m^{2} C_{p}^{2}}{16+9 C_{p}^{2}(1+d-m)^{2}}\right]\right\} \times \frac{3 C_{p}(1+d-m)}{\sqrt{16+9 C_{p}^{2}(1+d-m)^{2}}} \\
& \left.\times\left[\Phi\left(-\frac{9 m(1+d-m) C_{p}^{2}}{\sqrt{16+9 C_{p}^{2}(1+d-m)^{2}}}\right)-\Phi\left(-\frac{16+9(1+d)(1+d-m) C_{p}^{2}}{\sqrt{16+9 C_{p}^{2}(1+d-m)^{2}}}\right)\right]\right\} \\
& +\frac{K_{2}}{\Phi\left[3(1+d) C_{p}\right]+\Phi\left[3(1-d) C_{p}\right]-1}\left\{\Phi\left[3(1-d) C_{p}\right]-\Phi\left(3 n C_{p}\right)\right. \\
& -\left\{\exp \left[-\frac{72 n^{2} C_{p}^{2}}{16+9 C_{p}^{2}(1-d-n)^{2}}\right]\right\} \times \frac{3 C_{p}(1+d-n)}{\sqrt{16+9 C_{p}^{2}(1-d-n)^{2}}} \\
& \left.\left.\times\left[\frac{16+9(1-d)(1-d-n) C_{p}^{2}}{\sqrt{16+9 C_{p}^{2}(1-d-n)^{2}}}\right)-\Phi\left(\frac{9 n(1-d-n) C_{p}^{2}}{\sqrt{16+9 C_{p}^{2}(1-d-n)^{2}}}\right)\right]\right\}
\end{aligned}
$$

where $d$ denotes the difference between the target and the centre of specification divided by half of the specification width, $m$ denotes the difference between the target and the lower limit for the acceptable range, in which no quality loss will be incurred, divided by half of the specification width and $n$ denotes the difference between the target and the upper limit for the acceptable range, in which no quality loss will be incurred, divided by half of the specification width. Considering the scrap, rework and inspection costs, the total expected loss per unit product can be written as

$$
T C=L_{R I N L F}^{*}+\left[1-\Phi\left(3(1+d) C_{p}\right)\right] \times S C+\left[1-\Phi\left(3(1-d) C_{p}\right)\right] \times R C+I C
$$

Pan (2007) proposed a loss function-based risk assessment method by paring the process capability indices and loss functions. To demonstrate that various manufacturing and environmental risks can be evaluated by exploring the relationship between three loss functions and process capability indices, he illustrated the method using two numerical examples. However, the expected losses per unit product in determining the economic specification limits using different loss functions were not considered in his model.

When a process average is greater or less than the target value $T$, the derivation of the relationship between process capability indices and loss functions is similar to the case of the process average $\mu=T$, as described above.

\section{Selection of the appropriate loss function for economic tolerance design}

Based on the relationship between process capability indices and the total expected losses per unit product for various loss functions, the total expected losses per unit product for three loss functions under various $C_{p}$ can be compared. The total expected losses per unit product have been estimated under various $C_{p}$ values for both the symmetric and asymmetric tolerances when 


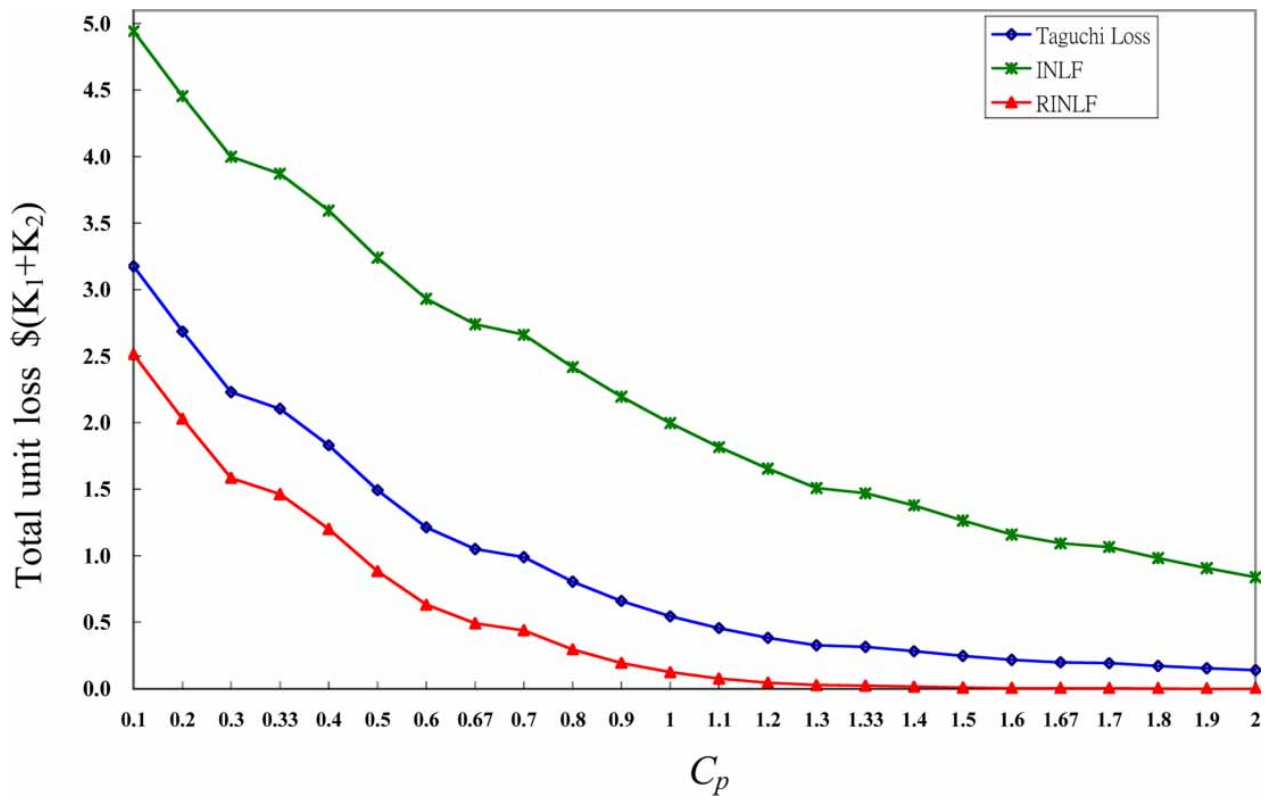

Figure 3. Comparison of the total expected losses per unit product for various loss functions under different $C_{p}$ (symmetric tolerance and symmetric loss case).

a process average $\mu$ is equal to $T$. Figure 3 shows that the total expected losses per unit product decline as the process capability index $C_{p}$ improves regardless of which loss function is used (here the case of symmetric tolerance and symmetric loss is illustrated as an example). Notice that the failure/defect rate is 66 p.p.m. (part per million) for $C_{p}=1.33$ or a four-sigma process, 0.54 p.p.m. for $C_{p}=1.67$ or a five-sigma process and 0.002 p.p.m. for $C_{p}=2$ or a six-sigma process (see Table 1 for details). When $C_{p}=2$, the total expected losses per unit product calculated using three different loss functions are $\$ 0.14\left(K_{1}+K_{2}\right)$ for the revised Taguchi quadratic loss function, $\$ 0.084\left(K_{1}+K_{2}\right)$ for the INLF loss function and \$0.00004 $\left(K_{1}+K_{2}\right)$ for the RINLF, respectively. Pan (2007) provided a new loss function-based method for evaluating manufacturing and environmental risks. Through two numerical examples he showed that the expected losses using Taguchi's loss function and the INLF are overestimated, especially for the four-, five- and six-sigma processes and the actual losses could be realistically reflected by the RINLF. Since engineering specification plays a key role in the calculation of $C_{p}$ or $C_{p k}$ and the loss estimation of the RINLF is more consistent with the defect rate than that of the revised Taguchi loss function and the INLF, it is suggested that the RINLF be used in the determination of economic specification limits.

\section{A numerical example}

Stencil printing is one of the most cost-effective processes for solder paste deposition and it has been widely used in traditional high-volume surface mount assembly. There are many variables that influence the quality of the stencil printing process, which are mainly measured by the amount and position of solder paste deposited. Instead of using the traditional way of setting the tolerance by $T \pm 5 \sigma$ in most electronic industries, the new economic specification limits are established based on the revised Kapur economic tolerance design model to minimize the product variation/loss shipped to the customer. To demonstrate how to determine the new economic specification limits using the RINLF, this article uses the solder volume on Quad Flat Package 
(QFP) pads deposited by a stencil of a thickness of $0.15 \mathrm{~mm}$ and aperture sizes of $0.5 \mathrm{~mm}$. The procedures of the new economic tolerance design are as follows.

\subsection{Economic tolerance design for the solder deposited volume of QFP pads}

In step 1 a statistical test is performed. The solder deposited volume data on 264 pads data were from experimental data (Pan et al. 2004). The data passed the normality test. The normal probability density function of the solder volume is shown in Figure 4, where the mean $\mu=$ $0.0507 \mathrm{~mm}^{3}$ and the standard deviation $\sigma=0.005 \mathrm{~mm}^{3}$.

Step 2. Determine RINLF for the solder deposited volume

The target of solder deposited volume $T=0.059 \mathrm{~mm}^{3}$. Assume the maximum quality loss per unit, $K_{1}=K_{2}=\$ 50$ (i.e. symmetric loss function) if the solder deposited volume is deviated from the target and exceeds the specification limits. Since solder paste applied to the board is washable and the board itself is reusable even if its deposited volume is out of the specification limits. Therefore, the scrap cost is not considered in the solder paste stencil printing process. Assume the rework cost $R C=\$ 20$ per unit if the solder deposited volume exceeds the specification limits and the inspection cost $I C=\$ 5$ per unit if $100 \%$ inspection is conducted. Suppose that the RINLF reaches its maximum loss at $T \pm 5 \sigma$ limits, where $T-5 \sigma=0.059-5 \times 0.005=0.034$ and $T+5 \sigma=0.059+5 \times 0.005=0.084$, no quality loss will be incurred if the solder deposited volume falls in the acceptable range $(L, U)=(0.0465,0.0715)$, where $L=0.059-0.0125=$ 0.0465 and $U=0.059+0.0125=0.0715$. Note that the parameters for controlling the shape of RINLF are: $\sigma_{L 1}=(0.0465-0.034) / 4=0.0031$ and $\sigma_{L 2}=(0.084-0.0715) / 4=0.0031$.

Step 3. Determine the economic specification limits for the solder deposited volume

Based on the revised Kapur economic tolerance design model, plug the parameter settings of RINLF from step 2 into Equation (2). Due to the fact that the board can be reworked if it exceeds specification limits, the total unit cost can be written as:

$T C=L_{R I N L F}^{*}+(1-q) \times R C+I C$. Moreover, the total unit cost TC is the function of $\eta_{1}$ and $\eta_{2}$, the mathematical equation for determining the new economic specification limits can be written as: $\operatorname{Min}\left\{\eta_{1}, \eta_{2}: T C\left(\eta_{1}, \eta_{2}\right)=L_{R I N L F}^{*}+(1-q) \times R C+I C\right\}$.

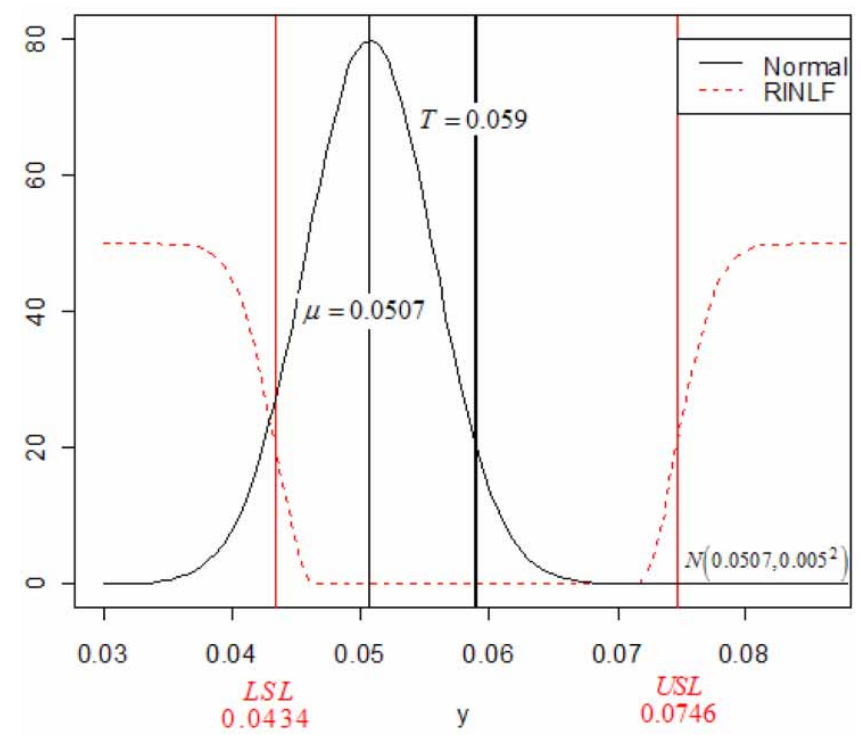

Figure 4. New $(L S L, U S L)$, RINLF and the probability density function $N(0.0507,0.0052)$. 
Table 2. Comparison of the economic tolerance design for solder deposited volume.

\begin{tabular}{lrcccr}
\hline & & & Economic lower limit & Economic upper limit \\
Loss Functions & \multicolumn{1}{c}{$\eta_{1}$} & $\eta_{2}$ & $L S L=0.0507-\eta_{1} \times 0.005$ & $\begin{array}{c}\text { USL=0.0507 }+\eta_{2} \times 0.005 \\
\text { Min }\{\mathrm{TC}\}\end{array}$ \\
\hline Revised Taguchi's & 1.9736 & 4.7250 & 0.0408 & 0.0743 & 12.3781 \\
INLF & -1.2409 & 2.0791 & 0.0569 & 0.0611 & 24.1814 \\
RINLF & 1.4653 & 4.7846 & 0.0434 & 0.0746 & 7.2421 \\
\hline
\end{tabular}

Table 3. The new economic tolerance design for solder deposited volume using RINLF.

\begin{tabular}{|c|c|c|c|c|c|c|c|c|}
\hline \multirow{2}{*}{$\begin{array}{l}\text { Quality } \\
\text { Characteristic }\end{array}$} & \multicolumn{3}{|c|}{ Parameters of process data } & \multicolumn{2}{|c|}{$\begin{array}{c}\text { Parameter Settings for RINLF } \\
\mathrm{K}_{1}=\mathrm{K}_{2}=\$ 50, R C=\$ 20, I C=\$ 5\end{array}$} & \multicolumn{2}{|c|}{$\begin{array}{l}\text { New economic specification } \\
\text { limits (LSL, USL) }\end{array}$} & \multirow[b]{2}{*}{$\operatorname{Min}\{\mathrm{TC}\}$} \\
\hline & $T$ & $\mu$ & $\sigma$ & $L, U$ & $\sigma_{L}$ & LSL & USL & \\
\hline $\mathrm{QFP}_{6 \mathrm{mil}, 20}$ & 0.059 & 0.0507 & 0.005 & $(0.0465,0.0715)$ & 0.0031 & 0.0434 & 0.0746 & $\$ 7.2421$ \\
\hline
\end{tabular}

One can solve the above equation using the attached $\mathrm{R}$ computer program (see Appendix for details) and achieve the optimal solution $\eta_{1}=1.4653, \eta_{2}=4.7846$ and $\operatorname{Min}\left\{\operatorname{TC}\left(\eta_{1}, \eta_{2}\right)\right\}=$ 7.2421. After plugging the optimal solution $\eta_{1}$ and $\eta_{2}$ into the formula $(L S L, U S L)=(\mu-$ $\left.\eta_{1} \sigma, \mu+\eta_{2} \sigma\right)$, then the new economic lower specification limit, $L S L-\mu-\eta_{1} \sigma=0.0507-$ $1.4653 \times 0.005=0.0434$ and the new upper specification limit, $U S L+\mu+\eta_{2} \sigma=0.0507+$ $4.7846 \times 0.005=0.0746$, where the range of $\eta_{1}$ and $\eta_{2}$ are:

$$
\begin{aligned}
\frac{\mu-L}{\sigma} & \leq \eta_{1} \leq \frac{\mu}{\sigma} \Longrightarrow \frac{0.0507-0.0465}{0.005} \leq \eta_{1} \leq \frac{0.0507}{0.005} \Longrightarrow 0.84 \leq \eta_{1} \leq 10.14 \\
\eta_{2} & \geq \frac{U-\mu}{\sigma} \Longrightarrow \eta_{2} \geq \frac{0.0715-0.0507}{0.005} \Longrightarrow \eta_{2} \geq 4.16
\end{aligned}
$$

The new economic specification limits ( $L S L, U S L)$, RINLF and the probability density function $N(0.0507,0.000025)$ for the volume of QFP pads are shown in Figure 4.

Notice that one can simply input the data into the attached $\mathrm{R}$ program to obtain new economic specification limits and minimum total costs using various loss functions even if the scrap cost and the rework cost are different for the case of asymmetric loss. Furthermore, similar procedures can be applied to obtain the economic specification limits for the solder deposited volume of QFP flat pads using the INLF and revised Taguchi loss functions. A comparison of the economic specification limits using the RINLF, INLF and revised Taguchi loss function as well as their respective total quality losses are summarized in Table 2.

It can be seen in Table 2 that the economic specification limits for the solder deposited volume of QFP flat pads using the RINLF has the minimum total unit cost and the new specification limits (0.0434 and 0.0746$)$ are the most realistic ones for monitoring the product quality of the solder deposited volume.

The basic statistics of the process data, parameter settings for the RINLF and the economic specification limits for the solder deposited volume are summarized in Table 3.

\section{Conclusions and future research}

This article proposes a new method for relaxing the limitation that the scrap and rework costs are the same (the case of symmetric loss). Six models were derived for estimating the total expected loss in determining the economic specification limits. The six models include three 
models for bilateral specification (the 'nominal the best' case) using the revised Taguchi quadratic loss function, the INLF and the RINLF and three models for the unilateral specifications (the 'smaller the better' case) using the three loss functions. To decide which loss function is the best in the economic tolerance design, the relationships between process capability indices and expected loss per unit under normal distribution were derived. The results suggest that the RINLF may be the most appropriate loss function in the economic tolerance design. Then, a comparative study of the economic tolerance design was conducted using the solder paste stencil printing process as an example. Although only the rework cost was considered in the numerical example, the results show that the new economic specification limits using the RINLF are the most realistic for monitoring the quality of solder deposited volume. One can easily extend it to the case of asymmetric loss (when the scrap cost is not equal to the rework cost) by simply changing the input parameters in the attached R program. Due to the fact that the true loss structure might not be symmetric in many situations, the use of symmetric loss function in determining new economic specification limits is not always recommended. Therefore, the asymmetric loss was considered in this article's models and it is suggested that RINLF be used in optimal symmetric and asymmetric tolerance design.

The above economic tolerance designs are based on the assumption that the quality characteristic follows a normal distribution. However, Cho and Phillips (1998) stated that the gamma distribution is more appropriate for the 'smaller-the-better' (S-type) quality characteristic and Chan et al. (2005) extended the cost model using loss functions for multiplying S-type quality characteristics. Research on the economic tolerance design under a non-normal distribution on the "nominal the best' (N-type) quality characteristics needs to be further explored.

\section{Acknowledgements}

The first author would like to gratefully acknowledge financial support from the National Science Council of Taiwan.

\section{References}

Barker, T.B., 1990. Engineering quality by design: interpreting the Taguchi approach. New York: Marcel Dekker.

Chan, L.K., Cheng, S.W. and Spiring, F.A., 1988. A new measure of process capability, $C_{p m}$. Journal of Quality Technology, $20(3), 162-175$.

Chan, W.M., Ibrahim, R.N., and Lochert, R.B., 2005. Quality evaluation model using loss function for multiple S-type quality characteristics. International Journal of Advanced Manufacturing Technology, 26 (1/2), 98-101.

Cho, B.R. and Phillips, M.D., 1998. Design of the optimum product specifications for S-type quality characteristics. International Journal of Production Research, 36 (2), 459-474.

Feng, Q. and Kapur, K.C., 2006. Economic development of specifications for $100 \%$ inspection based on asymmetric quality loss functions. IIE Transactions, 38 (8), 659-669.

Juran, J.M., 1974. Quality control handbook, 3rd edn. New York: McGraw-Hill.

Kane, V.E., 1986. Process capability indices. Journal of Quality Technology, 18 (1), 41-52.

Kapur, K.C., 1988. An approach for development of specifications for quality improvement. Quality Engineering, 1 (1), 63-77.

Kapur, K.C. and Cho, B.R., 1994. Economic design and development of specifications. Quality Engineering, 6 (3), 401-417.

Kapur, K.C. and Cho, B.R., 1996. Economic design of the specification region for multiple quality characteristics. IIE Transactions, 28 (3), 237-248.

Kapur, K.C. and Wang, C.J., 1987. Economic design of specifications based on Taguchi's concept of quality loss function. In: DeVor, R.E. and Kapoor, S.G., eds. Quality: design, planning, and control. Boston: The American Society of Mechanical Engineers, 23-36.

Leon, R.V. and Wu, C.F., 1992. A theory of performance measures in parameter design. Statistica Sinica, 2, 335-358.

Maghsoodloo, S. and Li, M.H., 2000. Optimal asymmetric tolerance design. IIE Transactions, 32 (12), 1127-1137.

Pan, J.N., 2007. A new loss function-based approach for evaluating manufacturing and environmental risks. International Journal of Quality and Reliability Management, 24 (8), 861-887.

Pan, J.N. and Li, Y.T., 2001. A study of loss functions for unilateral product specifications. Journal of Quality, 40 (1), 39-65. 
Pan, J.N. and Pan, J., 2006. A comparative study of various loss functions in the economic tolerance design. In: Proceedings of the 2006 IEEE International Conference on Management of Innovation and Technology, 21-23 June, Singapore, $783-787$.

Pan, J.N. and Wang, J.H., 2000. A study of loss functions for product interference analysis. Industrial Engineering Research, 2 (1), 80-100.

Pan, J., Tonkay, G.L., Storer, R.H., Sallade, R. and Leandri, D., 2004. Critical variables of solder paste stencil printing for micro-BGA and fine-pitch QFP. IEEE Transactions on Electronics Packaging Manufacturing, 27 (2), $125-132$.

Phadke, M.S., 1989. Quality engineering using robust design. New York: Prentice Hall.

Ryan, T.P., 1989. Statistical methods for quality improvement. New York: John Wiley \& Sons.

Spiring, F.A., 1993. The reflected normal loss function. The Canadian Journal of Statistics, 21 (3), 321-330.

Taguchi, G., 1986. Introduction to quality engineering: designing quality into products and processes. Tokyo: Asian Productivity Organization.

Tribus, M. and Szonyi, G., 1989. An alternative view of the Taguchi approach. Quality progress, 22 (5), 46-52.

\section{Appendix}

Listed below is the R program for solving the optimization models using various loss functions.

Solve the optimization model using the revised Taguchi's quadratic loss function.

$\mathrm{Q}<-$ function(eta1, eta2) pnorm(eta1)+pnorm(eta2) -1

Q1 <- function(eta1) 1-pnorm(eta1)

Q2 <- function(eta2) 1-pnorm(eta2)

EY <- function(eta1, eta2, mu, sigma) mu+(sigma/Q(eta1,eta2))*(dnorm(eta1)-dnorm(eta2))

VY <- function(eta1, eta2, mu, sigma) (sigma^2)*(1-(eta1/Q(eta1,eta2))*dnorm(eta1)-(eta2/Q(eta1,eta2))*dnorm(eta2)$\left((\text { dnorm(eta1)-dnorm(eta2))/Q(eta1,eta2) })^{\wedge} 2\right)$

A1 <- function(eta1, eta2, k1, k2) (k2-k1)/Q(eta1,eta2)

$\mathrm{A} 2<-$ function $\left(\mathrm{mu}, \mathrm{y} 0\right.$, sigma,eta2) $\left((\mathrm{mu}-\mathrm{y} 0)^{\wedge} 2+\operatorname{sigma}^{\wedge} 2\right)^{*}($ pnorm $($ eta2)-pnorm $((\mathrm{y} 0-\mathrm{mu}) /$ sigma $))$

A3 <- function(mu, y0, sigma) sigma*(mu-y0)*dnorm $((\mathrm{y} 0-\mathrm{mu}) /$ sigma $)$

A $4<-$ function $(\mathrm{mu}, \mathrm{y} 0$, sigma,eta 2$) 2 * \operatorname{sigma} *((\mathrm{mu}-\mathrm{y} 0)+($ eta $2 * \operatorname{sigma}) / 2) * \operatorname{dnorm}($ eta 2$)$

Lq <- function(mu, y0, sigma, k1, k2, eta1, eta2) $\{\mathrm{k} 1 *(\mathrm{VY}(\mathrm{eta} 1$, eta2, mu, sigma)+(EY(eta1, eta2, mu, sigma)$\left.\mathrm{y} 0)^{\wedge} 2\right)+\mathrm{A} 1(\mathrm{eta} 1, \mathrm{eta} 2, \mathrm{k} 1, \mathrm{k} 2)^{*}(\mathrm{~A} 2(\mathrm{mu}, \mathrm{y} 0$, sigma, eta2) +A3(mu, y0, sigma)-A4(mu, y0, sigma,eta2))

$\mathrm{TC}<-$ function $(\mathrm{mu}=10, \mathrm{y} 0=9.9$, sigma $=0.5, \mathrm{k} 1=5, \mathrm{k} 2=5, \mathrm{SC}=2, \mathrm{RC}=2, \mathrm{IC}=0.1)\{$ function $(\mathrm{eta}=\mathrm{c}(-1.658,1.658))\{\mathrm{Lq}(\mathrm{mu}$, $\mathrm{y} 0$, sigma, k1, k2, eta[1], eta[2])+Q1(eta[1])*SC+Q2(eta[2])*RC+IC \} \}

$\operatorname{optim}(\mathrm{c}((10-9.9) / 0.5-0.00001,(9.9-10) / 0.5)$, function(p) TC()(p))

Calculate and printout eta values and the minimum cost for the solder deposited volume.

TC <- function $\left(\mathrm{mu}=0.0507, \mathrm{y} 0=0.059\right.$, sigma $\left.=0.005, \mathrm{k} 1=50 /\left(\left(5^{*} 0.005\right)^{\wedge} 2\right), \mathrm{k} 2=50 /\left(\left(5^{*} 0.005\right)^{\wedge} 2\right), \mathrm{SC}=20, \mathrm{RC}=20, \mathrm{IC}=5\right)\{$ function $($ eta $=\mathrm{c}(-1.658,1.658))\{\mathrm{Lq}(\mathrm{mu}, \mathrm{y} 0$, sigma, $\mathrm{k} 1, \mathrm{k} 2$, eta[1], eta[2])+Q1 (eta[1])*SC+Q2(eta[2])*RC+IC $\}\}$ optim $(\mathrm{c}((0.0507-0.059) / 0.005-0.00001,(0.059-0.0507) / 0.005)$, function(p) TC ()$(\mathrm{p})$, lower $=c((0.0507-0.059) / 0.005-0.00001,(0.059-0.0507) / 0.005)$, upper $=c(0.0507 / 0.005$, Inf $))$

Solve the optimization model using the INLF and RINLF.

LSL <- function(mu, eta1,sigma) mu-eta $1 *$ sigma

USL <- function(mu, eta2,sigma) mu+eta2*sigma

$\mathrm{L}<-$ function(y0, delta1) y0-delta1

$\mathrm{U}<-$ function(y0, delta2) y0+delta2

$\mathrm{Q}<-$ function(eta1,eta2) pnorm(eta1)+pnorm(eta2) -1

Q1 <- function(eta1) 1-pnorm(eta1)

Q2 <- function(eta2) 1-pnorm(eta2)

A1L <- function(y0, delta1, mu, sigma) (L(y0, delta1)-mu)/sigma

A1U <- function(y0, delta2, mu, sigma) (U(y0, delta2)-mu)/sigma

$\mathrm{A} 2 \mathrm{~L}<-$ function(y0, delta1, mu, sigma, sigmal1) $\left((\mathrm{mu}-\mathrm{L}(\mathrm{y} 0, \text { delta1 }))^{\wedge} 2\right) /((\operatorname{sigma} 2+\operatorname{sigmal1} 2) * 2)$

A2U <- function $(y 0$, delta 2, mu, sigma, sigmal2 $\left.)((\text { mu-U(y0, delta2 }))^{\wedge} 2\right) /((\operatorname{sigma} 2+\operatorname{sigmal} 2 \wedge 2) * 2)$

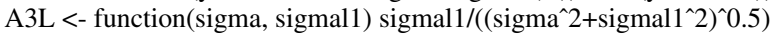

$\mathrm{A} 3 \mathrm{U}<-$ function(sigma, sigmal2) sigmal $2 /\left(\left(\operatorname{sigma} 2+\operatorname{sigmal} 2^{\wedge} 2\right)^{\wedge} 0.5\right)$

A4L <- function(y0, delta1, mu, sigma, sigmal1) ((L(y0, delta1)-mu)*sigmal1)/(sqrt(sigma^2+sigmal1^2)*sigma)

A4U <- function(y0, delta2, mu, sigma, sigmal2) ((U(y0, delta2)-mu)*sigmal2)/(sqrt(sigma^2+sigmal2^2)*sigma)

A5L <- function $\left(\mathrm{y} 0\right.$, delta1, mu, sigma, sigmal1, eta1) $\left((\text { mu-L }(\mathrm{y} 0, \text { delta1 }))^{*} \operatorname{sigma-eta} 1 *\left(\operatorname{sigma} 2+\operatorname{sigmal1}{ }^{\wedge} 2\right)\right) /(((\operatorname{sigma} 2$

+ sigmal1^2)^0.5)* sigmal1) 
A5U <- function(y0, delta2, mu, sigma, sigmal2, eta2) $\left((\mathrm{mu}-\mathrm{U}(\mathrm{y} 0, \text { delta2 }))^{*} \operatorname{sigma}+\right.$ eta $\left.2 *\left(\operatorname{sigma} 2+\operatorname{sigmal} 2{ }^{\wedge}\right)\right) /(((\operatorname{sigma} 2$ + sigmal2^2)^0.5)*sigmal2)

Lrinlf<- function(K1, K2, sigmal1, sigmal2, y0, mu, sigma, delta1, delta2, eta1, eta2) \{ (K1/Q(eta1,eta2))*(pnorm(A1L(y0, delta1, mu, sigma) $)+$ pnorm(eta1)-1-(exp(-A2L(y0, delta1, mu, sigma, sigmal1)) $)^{*} \mathrm{~A} 3 \mathrm{~L}($ sigma, sigmal1)*(pnorm(A4L(y0, delta1, mu, sigma, sigmal1))-pnorm(A5L(y0, delta1, mu, sigma, sigmal1, eta1))))+(K2/Q(eta1,eta2))*(pnorm(eta2)pnorm(A1U(y0, delta2, mu, sigma))-(exp(-A2U(y0, delta2, mu, sigma, sigmal2)) $* \mathrm{~A} 3 \mathrm{U}($ sigma, sigmal2)*(pnorm(A5U (y0, delta2, mu, sigma, sigmal2, eta2))-pnorm(A4U(y0, delta2, mu, sigma, sigmal2))) $\}$

TC <- function $(\mathrm{K} 1=50, \mathrm{~K} 2=50$, sigmal1 $=(3 * 0.005 * 1.67) / 4, \quad$ sigmal2 $=(3 * 0.005 * 1.67) / 4, \quad \mathrm{y} 0=0.059, \quad \mathrm{mu}=0.0507$, sigma $=0.005$, delta $1=0$, delta2 $=0, \mathrm{SC}=20, \mathrm{RC}=20, \mathrm{IC}=5)\{$ function $(\mathrm{eta}=\mathrm{c}(-1.658,1.658))\{$ Lrinlf $(\mathrm{K} 1, \mathrm{~K} 2$, sigmal1, sigmal2, y0, mu, sigma, delta1, delta2, eta[1], eta[2])+Q1(eta[1])*SC+Q2(eta[2])* RC+IC \}\}

Calculate and printout eta values and the minimum cost for the solder deposited volume using the INLF.

TC <- function $(\mathrm{K} 1=50, \mathrm{~K} 2=50$, sigmal $1=(3 * 0.005 * 1.67) / 4, \quad$ sigmal2 $=(3 * 0.005 * 1.67) / 4, \quad \mathrm{y} 0=0.059, \quad \mathrm{mu}=0.0507$, sigma $=0.005$, delta $1=0$, delta2 $=0, \mathrm{SC}=20, \mathrm{RC}=20, \mathrm{IC}=5)\{$ function $($ eta $=\mathrm{c}(-1.658,1.658))\{$ Lrinlf $(\mathrm{K} 1, \mathrm{~K} 2$, sigmal1, sigmal2, y0, mu, sigma, delta1, delta2, eta[1], eta[2])+Q1(eta[1])*SC+Q2(eta[2])*RC+IC \} \}

optim(c)((0.0507-0.059)/0.005-0.00001, (0.059-0.0507)/0.005), function(p) TC()(p),

lower=c((0.0507-0.059)/0.005-0.00001,(0.059-0.0507)/0.005), upper=c(0.0507/0.005,Inf $))$

Calculate and printout eta values and the minimum cost for the solder deposited volume using the RINLF.

TC <- function $(\mathrm{K} 1=50, \mathrm{~K} 2=50$, sigmal $1=(3 * 0.005 * 1.67 * 0.5) / 4$, sigmal2 $=(3 * 0.005 * 1.67 * 0.5) / 4, \mathrm{y} 0=0.059, \mathrm{mu}=0.0507$, sigma $=0.005$, delta $1=3 * 0.005 * 1.67 * 0.5$, delta $2=3 * 0.005 * 1.67 * 0.5, \mathrm{SC}=20, \mathrm{RC}=20, \mathrm{IC}=5)\{$

function $(\mathrm{eta}=\mathrm{c}(-1.658,1.658))\{$ Lrinlf $(\mathrm{K} 1, \mathrm{~K} 2$, sigmal1, sigmal2, y0, mu, sigma, delta1, delta2, eta[1], eta[2] $)+\mathrm{Q} 1(\mathrm{eta}[1])$ $* \mathrm{SC}+\mathrm{Q} 2(\mathrm{eta}[2]) * \mathrm{RC}+\mathrm{IC}\}\}$

$\operatorname{optim}(\mathrm{c}((0.0507-(0.059-3 * 0.005 * 1.67 * 0.5)) / 0.005,((0.059+3 * 0.005 * 1.67 * 0.5)-0.0507) / 0.005)$, function(p) TC ()$(\mathrm{p})$, lower $=\mathrm{c}((0.0507-(0.059-3 * 0.005 * 1.67 * 0.5)) / 0.005,((0.059+3 * 0.005 * 1.67 * 0.5)-0.0507) / 0.005)$, upper $=\mathrm{c}(0.0507 / 0.005$, Inf)) 